\title{
Efeito da intervenção multidisciplinar sobre a insatisfação da imagem corporal em ado- lescentes obesos
}

Rev Bras Ativ Fis Saúde p. 449-456 DOI: http://dx.doi.org/10.12820/23171634.2012v17n5p449

1 Programa Associado de Pós-graduação em Educação Física Universidade de Pernambuco/ Universidade da Paraiba, Recife, PE, Brasil.

\section{Effects of a multidisciplinary intervention on body image dissatisfaction in obese ad- olescents}

2 Programa de Pós-graduação em Nutrição, Universidade Federal de Pernambuco, Recife, PE, Brasil.

3 Escola Superior de Educação Física, Universidade de Pernambuco, Recife, PE, Brasil

4 Faculdade de Medicina, Universidade de Pernambuco, Recife, PE, Brasil.

Camila Rodrigues Menezes de Freitas

Mara Cristina Lofrano do Prado ${ }^{2}$

Priscyla Praxedes Gomes ${ }^{1}$

Nair Cristina Nogueira de Almeida ${ }^{3}$

Moacir de Novaes de Lima Ferreira ${ }^{4}$

Wagner Luiz do Prado ${ }^{1}$

\section{Resumo}

O objetivo do presente estudo foi analisar os efeitos da intervenção multidisciplinar sobre a insatisfação da imagem corporal em adolescentes obesos. Trinta e quatro adolescentes obesos com idade entre 12 a 19 anos, $\mathrm{IMC} \geq 95^{\text {th }}$ e púberes, foram submetidos à intervenção multidisciplinar composta por acompanhamento nutricional, psicológico, clínico e sessões de exercício físico. A insatisfação com a imagem corporal foi verificada através do Body Shape Questionnaire (BSQ). As avaliações foram realizadas antes e após 12 semanas de intervenção. Os efeitos da intervenção sobre a insatisfação da imagem corporal foram verificados utilizando-se o Qui-quadrado. Todas as análises foram realizadas com auxílio do software SPSS 10.0, p<0,05. Verificou-se no início da intervenção que $80,95 \%$ dos adolescentes apresentavam insatisfação com a imagem corporal, e após as 12 semanas de intervenção houve uma redução para 60,90\% dos adolescentes com algum grau de insatisfação com a imagem corporal $(p=0,05)$. Conclui-se que a intervenção multidisciplinar é uma importante estratégia para reduzir a insatisfação com a imagem corporal de adolescentes obesos.

\section{Palavras-chave}

Obesidade; Imagem corporal; Adolescentes.

\begin{abstract}
The aim of this study was to analyze the effects of a multidisciplinary intervention on body image dissatisfaction (BID) in obese adolescents. Thirty four obese adolescents aged between 12 to 19 years, $B M I \geq 95$ percentile and pubescent, were submitted to a multidisciplinary intervention composed by nutritional, psychological and clinical counseling and exercise sessions. BDI was determined assessed through by Body Shape Questionnaire (BSQ). Evaluations were performed before and after 12 weeks of intervention. The results are presented as mean $(X) \pm$ standard deviation (SD) and distribution of absolute and relative frequency. The effects of the intervention on $B D I$ were verified using chi-square test. All the analyses were performed using the software SPSS 10.0, $p<0.05$. A the beginning of intervention $80.95 \%$ of the adolescents were dissatisfied with their body image, after 12 weeks this value was reduced to $60.90 \%$ ( $p=0.05$ ). The multidisciplinary intervention is an important strategy to reduce the dissatisfaction with body image of obese adolescents.
\end{abstract}

\section{Keywords}

Obesity; Body image; Adolescents. 


\section{INTRODUÇÃO}

Imagem corporal é a figuração do corpo, construída pelo indivíduo em sua mente através do contato consigo mesmo e com o mundo ${ }^{1,2}$. Essa construção é multidimensional e descreve amplamente as representações internas da estrutura corporal e da aparência física ${ }^{3,4,5}$.

Indivíduos de ambos os gêneros, eutróficos ou obesos, podem apresentar insatisfação com a imagem corporal, porém derivada de perspectivas diferentes ${ }^{6}$. Pessoas obesas podem intensificar sua insatisfação a partir de comentários realizados por outras pessoas (ex. pais, amigos, companheiros), o que afeta diretamente sua auto-imagem e auto-estima. O simples fato de estar "acima do peso" é comumente associado à preguiça, falta de disciplina e falta de motivação, o que torna o obeso, muitas vezes, alvo de discriminação e bullying. A pressão para atingir o "corpo perfeito", preconizado pela sociedade atual, de um corpo magro para as mulheres e forte para os homens, aumenta a insatisfação com o próprio corpo, podendo levar a episódios de compulsão alimentar seguidos de tentativas mal-sucedidas de controle ponderal (especialmente via dietas restritivas) ${ }^{7}$.

Ironicamente, enquanto o "corpo ideal" continua a ser disseminado pela mídia e pelo discurso cultural, a prevalência de sobrepeso e obesidade aumenta, distanciando cada vez mais o indivíduo do ideal sociocultural ${ }^{8,9}$. A obesidade atinge números alarmantes em todo o mundo, estima-se que até 2015, aproximadamente 2,3 bilhões de adultos estarão com sobrepeso e mais de 700 milhões serão obesos ${ }^{10}$.

A obesidade afeta crianças, adolescentes e adultos ${ }^{10}$, de todos os estratos sociais ${ }^{8,9}$, no entanto, vale ressaltar que o início da adolescência é considerado um dos momentos mais críticos para o desenvolvimento desta doença, uma vez que nesta fase, mudanças físicas naturais do corpo se convertem em sentimentos de tensão sexual e atração física, influenciando os relacionamentos sociais e a reorganização de estruturas cognitivas relacionadas ao "corpo ideal" 11,12.

Enfatiza-se que programas de intervenção que incluam orientação nutricional, acompanhamento clínico, exercício físico e intervenções psicológicas são os mais indicados para o tratamento da obesidade ${ }^{13}$, e acrescentam que programas de intervenção são mais efetivos em crianças e adolescentes do que em adultos ${ }^{14}$.

Neste sentido, diversos estudos com adolescentes verificaram efeitos positivos desta abordagem terapêutica no controle síndrome metabólica ${ }^{15}$, esteatose hepática ${ }^{16}$ e qualidade de vida ${ }^{17}$, entretanto, até o presente momento poucos estudos avaliaram os efeitos da intervenção multidisciplinar na insatisfação da imagem corporal em adolescentes obesos, o que é essencial para se elevar as chances de sucesso do tratamento em longo prazo. Dessa forma, o objetivo do presente estudo foi analisar o efeito da intervenção multidisciplinar sobre a insatisfação da imagem corporal de adolescentes obesos.

\section{MÉTODOS}

Este estudo caracteriza-se como pré-experimental, descritivo e com abordagem quantitativa. Os voluntários foram recrutados por meio de anúncios em rádio e televisão da cidade do Recife e região metropolitana (julho/2010). O estudo foi aprovado pelo Comitê de Ética em Pesquisa da Universidade de Pernambuco (\#154/09), e todos os voluntários ou responsáveis legais assinaram termo de consentimento livre e esclarecido. 


\section{Amostra}

A amostra foi composta por 34 adolescentes obesos (16 meninos e 18 meninas) com idade entre 12 a 19 anos $(15,41 \pm 2,41$ anos). Os critérios de inclusão foram: Índice de Massa Corporal (IMC) $\geq 95$ percentil ${ }^{18}$, estágio maturacional púbere (3 e 4) de acordo com os critérios propostos por Tanner ${ }^{19}$ e motivação intrínseca (avaliada através de uma entrevista semi-estruturada conduzida pela psicóloga do grupo). Os critérios de exclusão foram: doença genética, metabólica ou endócrina (auto-relatada), consumo crônico de álcool, utilização prévia de drogas, e freqüência inferior a 75\% das sessões da intervenção (psicológica, nutricional, clínica e física).

\section{Avaliações}

As avaliações foram realizadas no início (pré) e após 12 semanas de intervenção (pós), sempre pelo mesmo avaliador e na mesma hora do dia, para se evitar quaisquer influências circadianas.

A massa corporal $(\mathrm{kg})$ foi determinada em uma balança Filizola, com escala de precisão de $0,1 \mathrm{Kg}$, a estatura foi aferida utilizando-se um estadiômetro fixo de madeira com escala de precisão de $0,1 \mathrm{~cm}{ }^{20}$. O IMC foi calculado dividindo-se a massa corporal pela estatura ao quadrado $\left(\mathrm{Kg} / \mathrm{m}^{2}\right)$. A composição corporal foi estimada por meio de impedância bioelétrica (Biodynamic Body Composition Analyser, modelo 310 - (Biodynamics Corporation, Seattle, EUA). A aptidão cardiorrespiratória (consumo máximo de oxigênio $\left(\mathrm{Vo}_{2 \max }\right)$ ) foi mensurada pela técnica de análise direta de gases, por meio de um protocolo incremental realizado em esteira rolante (InbrasportSuper ATL), conforme previamente descrito ${ }^{21}$.

Para avaliação da insatisfação da imagem corporal foi utilizado o Body Shape Questionnaire (BSQ), validado por Cooper ${ }^{22}$, traduzido para o Português ${ }^{23}$ e validado para a população brasileira ${ }^{24}$. O BSQé um questionário composto por 34 questões sobre insatisfação com a imagem corporal e preocupação com as medidas corporais, para cada questão deve ser atribuído um valor, em uma escala de 1 até 6 , sendo 1 (nunca) e 6 (sempre), o maior nível de insatisfação é dado pelo maior escore. Baseado na pontuação os indivíduos são classificados em satisfeitos (81 e 110 pontos) e insatisfeitos (acima de 111 pontos) com a imagem do corpo. $\mathrm{O}$ questionário foi aplicado pela psicóloga responsável, em uma sala calma e tranqüila, após os voluntários receberem todas as orientações.

\section{Intervenção Multidisciplinar}

O programa de tratamento multidisciplinar foi realizado durante 12 semanas, composto por acompanhamento clínico, físico, nutricional e psicológico (figura 1).

\section{Terapia Clínica}

A partir da primeira consulta com o endocrinologista responsável pelo projeto, os adolescentes receberam acompanhamento clínico mensal. Durante as consultas, o médico dava orientações sobre mudanças no estilo de vida e acompanhava a evolução clínica dos voluntários. Importante ressaltar que o médico não prescreveu qualquer medicação que pudesse alterar os resultados da presente pesquisa.

\section{Terapia Psicológica}

Foram realizadas sessões em grupo (aproximadamente 09 adolescentes), com uma hora de duração, conduzidas pela psicóloga do grupo. Durante as sessões foram abordados temas como emoções (sentimentos), autoestima, imagem corporal, preconceito, trans- 
tornos alimentares, dificuldades, questões familiares, bem como questões que pudessem surgir no grupo. Além disso, a psicóloga ficava à disposição dos voluntários para apoio e acolhimento para tratar de assuntos não relacionados à intervenção, se necessário.

\section{Terapia Nutricional}

A intervenção nutricional foi constituída por aulas de educação nutricional em pequenos grupos ( $\approx 10$ adolescentes), uma vez por semana, uma hora cada sessão, totalizando 12 sessões, abrangendo temas como pirâmide alimentar, dietas da moda, rotulagem nutricional, diferentes tipos de gordura, alimentação do tipo fast food, entre outros, ministradas sempre pela nutricionista responsável. Importante salientar que nenhuma dieta individualizada foi prescrita.

\section{Exercício Físico}

Os voluntários foram submetidos a um programa de exercício físico em esteira, supervisionado por educadores físicos, com duração de 60 minutos, três vezes por semana. A intensidade do exercício (entre $50 \%$ e $70 \%$ do $V_{o_{2 \max }}$ ) foi individualizada, baseada no $\mathrm{Vo}_{2 \max }$ obtido no teste incremental, todas as sessões tiveram gasto calórico fixado em $350 \mathrm{Kcal}$.

\section{Análise Estatística}

A análise estatística foi realizada com auxílio do software SPSS versão 10.0. A normalidade dos dados foi verificada através do Teste de Shapiro-Wilk. Os valores são apresentados em média $(\mathrm{X}) \pm$ desvio padrão $(\mathrm{DP})$. Diferenças entre os momentos pré e pós intervenção, para as variáveis antropométricas e composição corporal foram avaliadas através do Test $\mathrm{T}$ de Student para medidas dependentes. Os efeitos da intervenção sobre a insatisfação da imagem corporal foram verificados utilizando-se o Qui-quadrado. O nível de significância foi fixado em $\mathrm{p} \leq 0,05$.

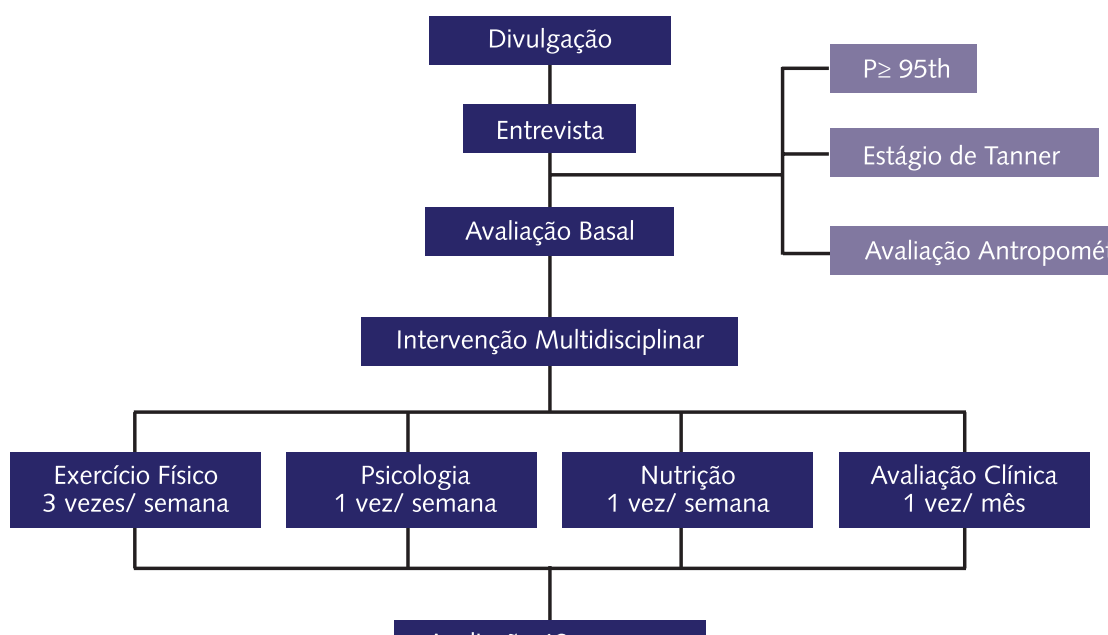

Avaliação 12 semanas

Figura 1 - Delineamento experimental.

$\mathrm{P}=$ Percentil.

\section{RESULTADOS}

No presente estudo, dos 34 adolescentes que iniciaram o estudo 21 voluntários (62\%) completaram a as 12 semanas de intervenção multidisciplinar com freqüên- 
cia superior a 75\% em todas as sessões nutricionais, psicológicas, clínicas e de exercício, sendo 10 meninas e 11 meninos.

$\mathrm{Na}$ tabela 1 são apresentados os dados antropométricos e composição corporal dos 21 adolescentes que completaram todos os procedimentos experimentais. Após a terapia verificou-se redução na porcentagem de gordura e massa gorda $(\mathrm{kg})$ e aumento na massa livre de gordura $(\mathrm{kg})$, entretanto, não foram encontradas diferenças na massa corporal total e IMC.

Em resposta a intervenção multidisciplinar houve redução ( $\mathrm{p}=0,000)$ no escore do BSQ de 109,38 $\pm 28,81$ para 103,00 $\pm 42,16$. No início do estudo $80,95 \%$ dos adolescentes apresentavam algum grau de insatisfação com a imagem corporal e, em resposta à intervenção, verificou-se diminuição no percentual de insatisfação para $60,91 \%$ ( $\mathrm{p}=0,05)$ (Figura 2). Não foram verificadas associações entre as alterações no BSQ com as variáveis antropométricas e de composição corporal.

Tabela 1 - Composição corporal e características antropométricas de adolescentes obesos submetidos à intervenção multidisciplinar $(\mathrm{N}=21)$.

\begin{tabular}{lccc}
\hline & Pré & Pós & \\
\hline Variável & $X \pm D P$ & $X \pm D P$ & $P$ \\
\hline Idade (anos) & $15,41 \pm 2,41$ & $15,60 \pm 241$ & 0,000 \\
\hline Estatura $(\mathrm{cm})$ & $164,6 \pm 6,52$ & $165,3 \pm 6,29$ & 0,015 \\
\hline MC $(\mathrm{kg})$ & $95,12 \pm 15,36$ & $95,04 \pm 14,06$ & 0,958 \\
\hline IMC $\left(\mathrm{kg} / \mathrm{m}^{2}\right)$ & $35,05 \pm 4,99$ & $34,45 \pm 4,61$ & 0,261 \\
\hline Gordura $(\%)$ & $39,23 \pm 13,18$ & $28,96 \pm 7,62$ & 0,000 \\
\hline MLG $(\mathrm{kg})$ & $57,53 \pm 14,31$ & $67,33 \pm 8,33$ & 0,002 \\
\hline MG $(\mathrm{kg})$ & $37,66 \pm 15,07$ & $28,16 \pm 9,26$ & 0,001 \\
\hline
\end{tabular}

Legenda: $M C=$ Massa Corporal; $I M C=$ Índice de Massa Corporal; $M L G=$ Massa Livre de Gordura; MG=Massa Gorda; $X=$ Médio; DP=Desvio Padrão.

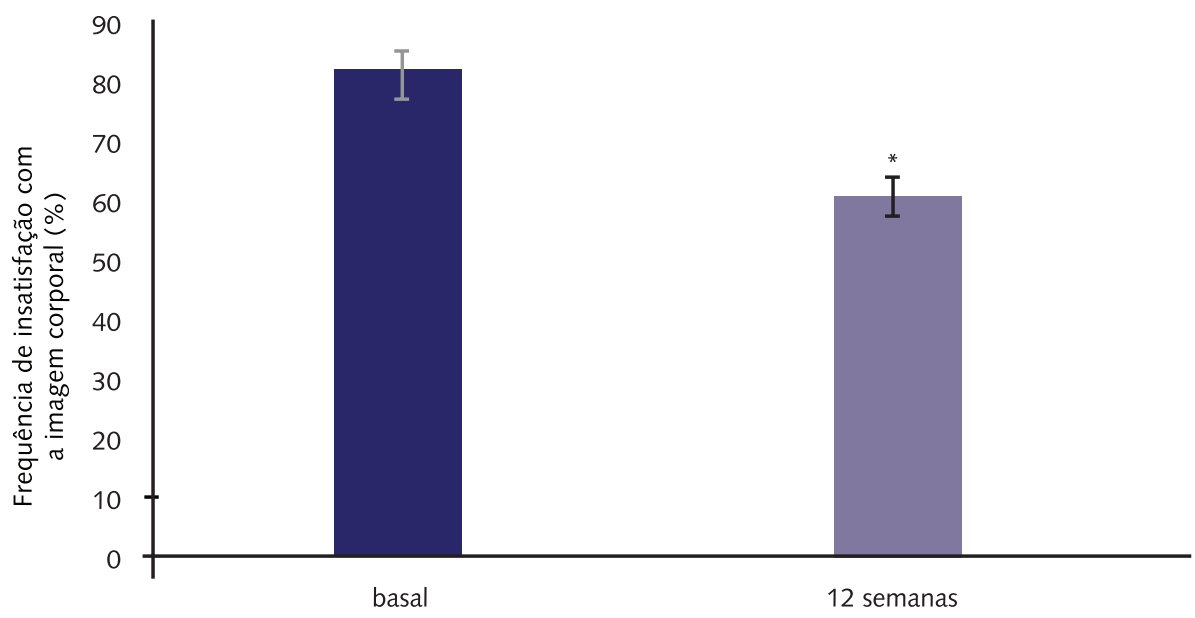

Figura 2 - Insatisfação com a imagem corporal de adolescentes obesos submetidos à intervenção multidisciplinar. ${ }^{*} \mathrm{p}=0,05$.

\section{DISCUSSÃO}

A obesidade está relacionada a problemas psicológicos, frustrações, infelicidade, além de uma gama de doenças físicas ${ }^{5}$. O excesso de peso tem forte ligação com a insatisfação corporal, baixa auto-estima, o que aumenta a incidência de com- 
portamentos inadequados (alimentares e físicos) ${ }^{17,25}$, destaca-se que esses fatores figuram entre as principais barreiras para o tratamento da obesidade.

A insatisfação com a imagem corporal tem sido associada ao desenvolvimento de transtornos alimentares, como a anorexia e a bulimia ${ }^{17}$, e de casos subclínicos caracterizados por comportamentos inadequados, como o uso de laxantes e diuréticos, autoindução de vômitos e excesso de exercícios físicos ${ }^{6}$. Além disso, as preocupações com o corpo e com a aparência física também podem causar prejuízos psicológicos, como baixa autoestima e depressão, prejudicando o desempenho físico, psicossocial e cognitivo do indivíduo.

A obesidade pode ser influenciada por fatores biológicos, psicológicos e sócio -econômicos, gerando seqüelas no indivíduo acometido pela doença. No âmbito psicológico problemas psicossociais como discriminação, bullying e falta de aceitação pelas pessoas podem resultar em graves conseqüências psíquicas tais como, isolamento, afastamento das atividades sociais e depressão ${ }^{5,7,25}$.

Nossos resultados demonstraram grande insatisfação dos adolescentes obesos com a sua imagem corporal no início do tratamento, provavelmente devido ao modelo de beleza imposto pela mídia, ser magro na sociedade atual é sinônimo de beleza e sucesso ${ }^{26}$. A intervenção multidisciplinar proporciona ao indivíduo a percepção de ser capaz da prática de exercício físico, melhoria das condições alimentares, relacionamentos sociais mais sólidos, resultando em alterações positivas na qualidade de vida ${ }^{17}$. Apesar das meninas expressarem maior insatisfação com a imagem corporal do que os meninos, ambos os gêneros sentem insatisfação com a imagem do corpo, no entanto, eles o experimentam de forma diferente, o que talvez possa ser explicado pela cultura que denominam mulheres magras e homens fortes ${ }^{27}$.

Destaca-se que na adolescência, o indivíduo enfrenta, além das transformações psicológicas próprias desta fase, mudanças biológicas naturais que ocorrem no corpo. É através do corpo que o adolescente se comunica com as outras pessoas e busca seu espaço, identificação e aceitação no mundo, desta forma, ter uma imagem corporal diferente do que é proposto pelo ideal de beleza (um corpo magro) faz com que o adolescente se sinta excluído e passe a rejeitar o próprio corpo ${ }^{28}$.

$\mathrm{O}$ culto à magreza intensifica o afastamento da pessoa obesa do convívio social ${ }^{8,9}$, o que pode, pelo menos em parte, explicar a evasão de $38 \%$ dos adolescentes observada no presente estudo, advindo em alguns casos da baixa auto-estima, constituindo uma das maiores dificuldades para a adesão no tratamento da obesidade em adolescentes.

Os jovens em geral, e não apenas os obesos, são insatisfeitos com seu corpo ${ }^{6}$. Avaliando 1.807 estudantes, com idade entre 7 e 19 anos, e observaram que 59\% desses indivíduos eram insatisfeitos com a imagem corporal e $56 \%$ praticavam atividade física com o objetivo de perder peso ${ }^{6}$, ainda neste sentido, outro estudo avaliou escolares de 8 a 10 anos e encontrou uma alta prevalência de insatisfação corporal $(69,9 \%)$, sendo que "apenas" $16,9 \%$ apresentavam sobrepeso ${ }^{29}$.

No presente estudo, ao contrário dos supracitados, aos adolescentes avaliados encontravam-se no mesmo estágio maturacional, o que minimiza vieses relacionados ao desenvolvimento sexual dos indivíduos. Por outro lado, a utilização de dobras cutâneas para estimativa da composição corporal, pode ser considerada uma fragilidade do estudo. Vale ressaltar que, o desenho experimental utilizado não permite quantificar a colaboração específica de cada uma das intervenções para a redução da imagem corporal nestes pacientes, devendo este ponto ser considerado na interpretação e aplicação dos resultados. 
Em resposta à intervenção multidisciplinar houve redução da insatisfação com a imagem corporal, mesmo sem redução na massa corporal e IMC, ou seja, os voluntários do presente estudo continuaram com a massa corporal acima da recomendada (ou preconizada). Neste sentido, podemos especular que adolescentes obesos satisfeitos com o seu corpo, possuem um auto conceito positivo e elevada auto-estima, influenciando de forma positiva o comportamento e as vivências do indivíduo ${ }^{30}$. A auto-estima e auto conceito positivos elevam o bem estar psicológico individual, levando o indivíduo a sentir mais confiança em si mesmo, e acreditando que é capaz de enfrentar todas as dificuldades. Isso torna o adolescente mais motivado a aderir a programas de intervenção, especialmente em longo prazo.

\section{CONCLUSÃO}

Conclui-se que a intervenção multidisciplinar, mesmo sem promover alterações na massa corporal e grau de obesidade, é capaz de aumentar a satisfação em relação à imagem corporal em adolescentes obesos, o que, em longo prazo, pode gerar efeitos benéficos tanto na saúde física quanto emocional destes pacientes.

\section{REFERÊNCIAS}

1. Almeida GAN, Santos JE, Pasian SR, Loureiro SR. Percepção de tamanho e forma corporal de mulheres: estudo exploratório. (Perceptions of body shape and size in women: an exploratory study.). Psicol Reflex Crit 2005; 10:27-35. doi: 10.1590/S1413-73722005000100005.

2. Schilder PF. The Image and the Appearance of the Human Body: Studies in Constructive Energies of the Psyche. London: Trench e Trubner; 1935.

3. Cash TF. Body image: Past, present, and future. Body Image 2004; 1:1-5. doi:10.1016/S17401445(03)00011-1.

4. Slade P. What is Body Image? Behav Res Ther 1994; 32:497-502. doi:10.1016/00057967(94)90136-8.

5. Masset KVSB, Safons MPS. Excesso de peso e insatisfação com a imagem corporal em mulheres. (Weight excess and dissatisfaction with body image in women.). Arq Sanny Pesq Saude 2008; 1:38-48. Retirado de http://www.cepsanny.com.br/pdf/v1n1a6.pdf.

6. Vilela J, Lamounier JA, Filho Dellaretti MA, Neto Barros J R, Horta GM. Transtornos alimentares em escolares. (Eating disorders in school children.).J Pediatr 2004; 80: 49-54. doi: 10.1590/S0021-75572004000100010.

7. Alves D, Pinto M, Alves S, Mota A, Leirós V. Cultura e imagem corporal. (Culture and body image). Motri 2009; 5:1-20. Disponível em: http://www.scielo.oces.mctes.pt/scielo. php?pid=S1646-107X2009000100002\& script=sci_arttext.

8. Ogden CL, Carroll MD, Curtin LR, McDowell MA, Tabak CJ, Flegal KM. Prevalence of overweight and obesity in the United States, 1999-2004. J Am Med Assoc 2006; 295:15491555. doi: 10.1001/jama.295.13.1549.

9. Damasceno VO, Vianna VRA, Vianna JM, Lacio M, Lima JRP, Novaes JS. Imagem corporal e corpo ideal. (Body image and ideal body.). Rev Bras Ciênc e Mov 2006; 14:87-96. Disponível em: http://portalrevistas.ucb.br/index.php/RBCM/article/viewArticle/691.

10. World Health Organization. Health topics: Obesity. WHO, 2010. Recuperado em 30 de setembro, 2010 de http://www.who.int/topics/obesity/en.

11. Borzekowski DLG, Bayer AM. Body image and media use among adolescents. Adolescent Med Clin 2005; 16:289-313. doi:10.1016/j.admecli.2005.02.010.

12. Fonseca MHR. Obesidade na adolescência: Um contributo para a melhor compreensão dos fatores psicossociais associados à obesidade e excesso de peso nos adolescentes portugueses. (Obesity in adolescence: A contribution for the best comprehension of the psychosocial factors associated to the obesity and excess weight in the Portuguese adolescents.). (tese de doutorado). Lisboa (Portugal): Faculdade de Medicina da Universidade de Lisboa; 2008. 
13. Curioni CC, Lourenço PM. Long-term weight loss after diet and exercise: a systematic review. Int J Obes 2005; 29:1168-1174. doi:10.1038/sj.ijo.0803015.

14. Snethen JA, Broome ME, Cashin S. Effective weight loss for overweight children: A metaanalysis of intervention studies.J Pediatr Nurs 2006;21:45-56. doi:10.1016/j.pedn.2005.06.006.

15. Caranti DA, de Mello MT, Prado WL, Tock L, Siqueira, KO, de Piano A et al. Short- and longterm beneficial effects of a multidisciplinary therapy for the control of metabolic syndrome in obese adolescents. Metabolism 2007; 56:1293-300. doi:10.1016/j.metabol.2007.05.004.

16. Piano A, Prado WL, Caranti DA, Siqueira KO, Stella SG, Lofrano-Prado MC et al. Metabolic and Nutritional Profile of Obese Adolescents With Nonalcoholic Fatty Liver Disease.J Pediatr Gastroenterol Nutr 2007; 44:446-452. doi: 10.1097/MPG.0b013e31803815d9.

17. Lofrano-Prado MC, Antunes HKM, Prado WL, Piano A, Caranti DA, Tock L et al. Quality of life in Brazilian obese adolescents: effects of a long-term multidisciplinary lifestyle therapy. Health Qual Life Outcome 2009; 7:61. doi: 10.1186/1477-7525-7-61.

18. Must A, Dallal GE, Dietz WH. Reference data for obesity: 85 th and 95 th percentiles of body mass index (wt/ht2) and triceps skin fold thickness. Amer J Clin Nutr 1991; 53:839-846. Disponível em: http://www.ajcn.org/content/53/4/839.long.

19. Tanner JM, Whithouse RH. Clinical Longitudinal standards for height, weight, weight velocity and stages of puberty. Arch Dis Child 1976; 51:170-179. doi:10.1136/adc.51.3.170.

20. Lohman TG, Roche AF, Martorrel R. Anthropometrics Standardization Reference Manual 1988 Champaign, Illions, Human Knectis.

21. Mcconnell TR. Practical Considerations in the testing of O2max in runners. Sports Med 1988; 5:57-68. Disponível em: http://adisonline.com/sportsmedicine/toc/1988/05010.

22. Cooper PI, Taylor MJ, Cooper Z, Fairbun CG. The development and validation of the Body Shape Questionnaire. Int J Eat Disord 1987; 6:485-94. doi: 10.1002/1098-108X(198707)6:4<485::AID-EAT2260060405>3.0.CO;2-O.

23. Cordás TA. Questionário de Imagem Corporal: versão para mulheres. (Body Image questionnarie: woman version.). In C. Gorestein, L. H.; Andrade, S. G., \& A. W. Zuardi (Eds.), Escalas de avaliação clínica em psiquiatria e psicofarmacologia. São Paulo: Lemos Editorial, 2000. p. 352-353.

24. Manetta MCDP. Validade interna, dimensionalidade e desempenho da escala BSQ "Body Shape Questionaire” em uma população de estudantes universitários. (Internal validity and performed of scale BSQ "Body Shape Questionaire" in a population of university students.). (dissertação de mestrado). São Paulo (SP): Universidade Federal de São Paulo; 2002.

25. Lima JRP, Orlando FB, Teixeira MP, Castro APA, Damasceno VO. Conjunto de silhuetas para avaliar a imagem corporal de participantes de musculação. (Silhouettes for evaluate the body image of participants of weight training.). Arq Sanny Pesq Saúde 2008; 1:26-30. Disponível em: http://www.cepsanny.com.br/pdf/v1n1a4.pdf.

26. Ricciardelli LA, McCabe MP, Banfield S. Sociocultural influences on body image and body changes methods. J Adolesc Health 2000; 26: 3-4. doi:10.1016/S1054-139X(99)00107-X.

27. Neighbors LA, Sobal J. Prevalence and magnitude of body weight and shape dissatisfaction among university students. Eat Behav 2007; 8:429-439. doi:10.1016/j.eatbeh.2007.03.003.

28. Olmsted MP, McFarlane T. Body Weight and Body Image. BMC Womens Health 2004; 4. S5. doi: 10.1186/1472-6874-4-S1-S5.

29. Triches RM, Giuglian ERJ. Insatisfação corporal em escolares de dois municípios da região Sul do Brasil. (Body dissatisfaction in school children from two cities in the South of Brazil.). Rev Nutr 2007; 20:119-128. doi: 10.1590/S1415-52732007000200001.

30. Assis SG, Avanci JQ, Silva CMFP, Malaquias JV, Santos NC, Oliveira RVC. A representação social do ser adolescente: um passo decisivo na promoção da saúde. (The social representation to be adolescent: a decisive step to health promotion.). Ciênc Saúde Coletiva 2003; 8:669-680. doi: 10.1590/S1413-81232003000300002.

$$
\begin{array}{r}
\text { Endereço para Correspondência } \\
\text { Wagner Luiz do Prado. } \\
\text { Escola Superior de Educação Física- } \\
\text { (ESEF/UPE), Rua Arnóbio Marques, } 310 \\
\text { Santo Amaro, Recife- PE. } \\
\text { CEP: } 50.100 .130 . \\
\text { Campus Universitário HUOC - ESEF. } \\
\text { Telefone/Fax: } 81-31833373 . \\
\text { E-mail : wagner.prado@upe.br } \\
\text { wagner.prado@pq.cnpq.br }
\end{array}
$$

Recebido 06/11/2012

Revisado 19/01/2013

Aprovado 25/01/2013 